\title{
Rocker Outsole Shoes and Margin of Stability during Walking: a Preliminary Study
}

\author{
Banafshe Ghomian \\ Prosthetics \& Orthotics Department Iran University of \\ Medical Sciences \\ Tehran, Iran \\ Sina Mehdizadeh \\ Biomechanics and Performance Analysis Department \\ National Sports Institute \\ Kuala Lumpur, Malaysia \\ Rokhsareh Aghili \\ Institute of Endocrinology and Metabolism Iran \\ University of Medical Sciences Tehran, Iran
}

\author{
Roozbeh Naemi \\ Faculty of Life Sciences and Education Staffordshire \\ University Staffordshire, UK \\ Hassan Jafari Department of Health Psychology \\ University of Leuven \\ KU Leuven, Belgium
}

José Machado, Luís F. Silva, Pedro Lobarinhas Mechanical Engineering Department University of Minho

Guimarães, Portugal

\author{
Hassan Saeedi \\ Prosthetics \& Orthotics Department Iran University of Medical Sciences \\ Tehran, Iran
}

\begin{abstract}
Rocker outsole shoes are commonly prescribed to diabetic patients to redistribute peak plantar pressures at high risk areas such as forefoot thereby decreasing risks of plantar ulceration. However, there are evidences of postural destabilizing effect of the rocker outsole shoes. Patients with diabetes, particularly those suffering from neuropathy, are at a higher risk of falling. Then, beside primary role of rocker outsole in offloading, it is essential to explore the effect of the rocker outsole on postural stability of these patients. The smaller base of support (BOS) of the rocker outsoles due to their specific geometry has been previously mentioned as a threat to postural stability. Margin of stability (MOS) appeared to be an appropriate method for measuring postural stability during walking by considering the dynamicity of BOS in its equation. MOS is defined as the distance between the velocity adjusted position of the centre of mass (COM) and the boundaries of the BOS which constantly changes during walking. Then, this study proposes how we can use MOS concept to investigate the impact of the rocker outsoles on dynamic stability. In this study the kinematic data from a single subject with diabetic neuropathy was used to explain the efficiency of the methodology. Conclusive results are expected after carrying out systematic tests using a statistically representative sample size.
\end{abstract}

Keywords - rocker outsole; dynamic stability; margin of stability; centre of mass; diabetic neuropathy

This project is funded by Iran University of Medical Sciences (IR.IUMS.REC. 1395.9211503202).

\section{INTRODUCTION}

Diabetes Mellitus is one of the most prevalent metabolic disorders around the world. There were almost around 381.8 million people with diabetes in the year 2013 and more than

$45.8 \%$ of them were clinically undiagnosed [1]. Diabetic foot is one of the most serious complications and a major risk factor for foot ulcerations with life time risk of developing of approximately $15-25 \%$ among diabetic patients [2], [3]. If diabetic ulcer left untreated or not fully treated, it might lead to a limb loss. Almost $85 \%$ of lower extremity amputations in patients with diabetes have been reported to occur following foot ulcerations [4]. To prevent plantar foot ulceration and its further complications, management of pressure distribution at high-risk areas, such as the forefoot is essential [5], [6]. Rocker outsoles are currently the most effective intervention capable of reducing forefoot peak plantar pressure by up to $50 \%$ [7]-[9].

Another life-threatening consequences of diabetes is postural instability which can potentially increase the risks of falling and further trauma [10]-[12]. Patients with diabetes have an altered gait pattern which has been corresponded to either their muscle strength alteration due to diabetes [13], [14] or a compensatory strategy as an effort to decrease pick plantar pressures [15], [16]. Moreover, up to $50 \%$ of people with diabetes suffer from neuropathy [17] that cause them to be 
prone to fall approximately five times more than the healthy people [18]-[20].

Besides these, there are several evidences of postural destabilizing effect and thus likelihood of falling of the rocker outsole shoes on both healthy and diabetic people [21]-[23]. Although it has been frequently mentioned that most falls occur during walking [24], [25], these studies only investigated the static (bipedal stance) or quasi-dynamic (perturbed stance) postural stability while wearing the rocker outsole shoes. As the main goal of the rocker outsole is to offload the foot during walking, it is much more important to investigate its impacts during gait. However, the main focus of such studies have been on the rocker outsole capability to redistribute pressure and not on the dynamic postural stability. Therefore, there is a paucity of research about the impacts of the rocker outsole shoe on dynamic stability of patients with diabetic neuropathy during walking.

Rocker outsole shoes have a smaller BOS compared to either barefoot or typical shoe outsoles, providing a smaller contact area with the ground at each instant of walking [21]. Moreover, the specific geometry of the rocker outsole facilitates the roll-over function during terminal stance, however it speeds up the propulsion phase that may affect stability [26]. Therefore, it would be worthwhile if we could explore the impact of the rocker outsole shoes on dynamic stability during walking with regard to the BOS.

Static stability is defined as an ability to maintain the vertical projection of the COM within the BOS polygon while standing upright [27]. However, this definition cannot be properly applied to dynamic conditions such as walking without considering the horizontal COM velocity as well as the instantaneously changing BOS polygon. In order that this definition could be applied to the dynamic situations, Hof et al. proposed a new concept termed as 'margin of stability' (MOS) [28]. The distance between the velocity adjusted COM position termed 'extrapolated' position of COM (XcoM) and the anteroposterior or medio-lateral boundaries of the instantaneous BOS during walking defines the MOS. This definition further suggests that an individual can alter either the positioning of the foot or the COM motion in order to control the margin of stability [29]. This might propose a probable aim of maintaining some minimum MOS during walking in order to remain stable.

In this study, we showed how the previously proposed method of MOS can be applied to investigate dynamic stability of a patient with mild diabetic neuropathy while walking with different rocker outsole shoes. Also, we tried to make some statistical comparisons between different conditions to explain the results.

\section{Methods}

\section{A. Subject}

One diabetic female with ten years of diabetic duration and a clinically diagnosed mild neuropathy, through using Michigan Neuropathy Screening Index (MNSI) followed by Nerve Conduction Velocity (NCV) test, aged 50 years and body mass index of $30.1(\mathrm{~kg} / \mathrm{m} 2)$, without any history of previous ulceration, falls or neurological/musculoskeletal disorders was recruited to the study after the ethical approval was granted by the local university. A written consent was also obtained from the participant.

\section{B. Experimental Protocol}

Three different (A, B and C) designs of toe-only rocker outsoles were used - with the rocker angle, apex angle, apex position ( $\%$ of the shoe length from heel) Fig. 1 and heel height - as follows:

$$
\begin{aligned}
& \text { A: } 10^{\circ}, 80^{\circ}, 60 \%, 2 \mathrm{~cm} ; \\
& \text { B: } 15^{\circ}, 95^{\circ}, 52 \%, 3.5 \mathrm{~cm} ; \\
& \text { C: } 20^{\circ}, 95^{\circ}, 60 \%, 4 \mathrm{~cm} .
\end{aligned}
$$

These designs were previously shown to be most effective in plantar pressure reduction in diabetic patients [30]. All experimental shoes used in this study were provided from the same brand, made from cotton and had a straight last and $3 \mathrm{~mm}$ thickness. Also, all rocker outsoles were made from microcell with the standard hardness of 53 in class A shore scale. Rigid steel shanks with $1 \mathrm{~mm}$ thickness and $2.5 \mathrm{~cm}$ width and an equal length for each rocker shoe, were attached to the midsole of the shoes for rocker B and C, and not for the rocker A, as it is mostly resembling a typical rocker outsole commonly used in typical shoes. Motion data from 44 reflective markers were used to record full body kinematics using 6 infrared cameras sampling at $100 \mathrm{~Hz}$ applying Plug-in gait model (Qualisys Track Manager, QTM, Sweden) while the subject walked with a self- selected speed over a 10-meter walkway in the Gait Analysis laboratory of the local university. After a 5-minute adaptation time to each of three rocker outsole shoes while the subject walked in a handrail for safety purposes, she was asked to complete three successful walking trials with each of the three rocker outsole shoes and barefoot. The order at which the rocker outsole shoes were used was randomized to minimize the learning effect.
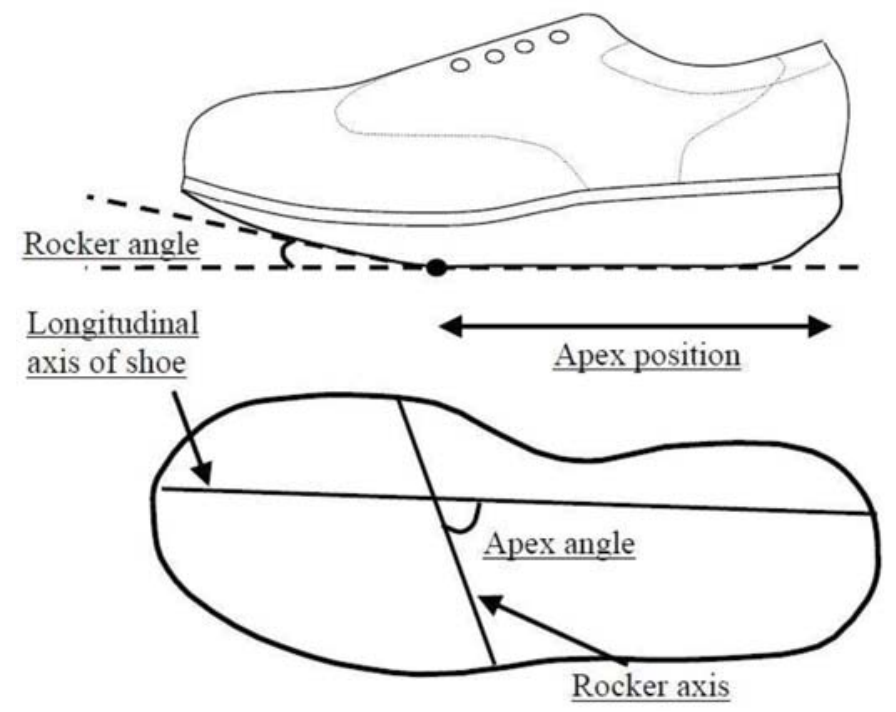

Fig. 1 Three characteristics of a rocker outsole [30] 


\section{Data Analysis}

Gaps in motion data were filled by a spline interpolation using Matlab R2010a (Math works Inc., Natick, MA). Then, whole body COM was computed based on a 15-segment model specifically created for the subject based on her anthropometric data in Visual 3D software. Heel strikes were determined by using the minimum value for the vertical position of the heel marker. MOS was calculated as [28]:

$$
\begin{gathered}
X C O M=C O M+\frac{C O M}{\omega_{0}}, \omega_{0}=\sqrt{g / l} \\
M B O S=B O S-X C O M
\end{gathered}
$$

Where $C O M$ and $C \dot{O} M$ are the position and velocity of the $\mathrm{COM}$ in either antero-posterior or medio-lateral directions, $g=9.81 \mathrm{~m} / \mathrm{s} 2$ is the gravitational constant and $l$ is the distance between the COM and the lateral heel marker at heel strike ( $\approx$ leg length).

The anteroposterior border of BOS was defined by the anteroposterior position of the toe marker and was used for the calculation of the anteroposterior MOS (MOSap). The mediolateral border of BOS was defined as the medio-lateral position of the lateral rocker marker (which was placed exactly on the lateral aspects of the rocker apex for shoed conditions and on the 5th metatarsal head for the barefoot condition) and was used to calculate the medio-lateral MOS (MOSml). MOSap and MOSml were calculated for each step at each heel strike as it was previously shown that MOS reaches its minimum value almost at heel strike. Moreover, MOS was calculated such that positive values indicated stability (i.e. when XcoM was inside the BOS) and negative values indicated instability (i.e. when XcoM was outside of the BOS). Also, for MOSml equation 2 could be defined as $\mathrm{MOS}=\mathrm{XCOM}-\mathrm{BOS}$ depending on choice of the foot (left or right leg side) analyzed [31].

\section{STATISTICS}

A mixed effect model followed by Student's t pairwise comparisons was applied to compare four conditions with regards to the choice of the foot (i.e. the first and second subsequent step). Choice of the foot and condition were considered as the independent variables and the trial was considered as a random effect. Statistical analyses were performed using JMP software (SAS Institute Inc., USA).

\section{RESUlts}

Mean MOSap over trials was significantly different for barefoot condition compared to all three rocker outsole conditions $(p<0.05)$ so that it had a positive value while others were negative and also was lower in magnitude than all three rocker outsole conditions TABLE I. Mean MOSap over trials for rocker outsole A was significantly greater than both the rocker outsoles B and C. No significant difference was found for mean MOSap between rocker outsole B and C Fig. 2. Also, the effect of choice of the foot for MOSap was not significant $(\mathrm{p}>0.05)$.
Table I The results of MOSap Multiple Comparisons for Condition (Student's t All Pairwise Comparisons

\begin{tabular}{l|l|l|l|l|}
\hline Condition & -Condition & Difference & Std Error & Prob $>|\mathbf{t}|$ \\
\hline Barefoot & Rocker A & 462.247 & 74.89967 & $<.0001^{*}$ \\
\hline Barefoot & Rocker B & 666.033 & 73.23840 & $<.0001^{*}$ \\
\hline Barefoot & Rocker C & 641.092 & 79.59514 & $<.0001^{*}$ \\
\hline Rocker A & Rocker B & 203.787 & 68.41026 & $\mathbf{0 . 0 0 6 8 *}$ \\
\hline Rocker A & Rocker C & 178.845 & 74.89967 & $\mathbf{0 . 0 2 6 0 *}$ \\
\hline Rocker B & Rocker C & -24.942 & 73.23840 & 0.7367 \\
\hline
\end{tabular}

The symbol * indicates significance of the difference.

Moreover, no significant differences were found for mean $\mathrm{MOS}_{\mathrm{ml}}$ among all conditions ( $\left.\mathrm{p}>0.05\right)$ TABLE II, but the effect of the choice of the foot for $\operatorname{MOS}_{\mathrm{ml}}$ was significant $(\mathrm{p}<0.0001)$.

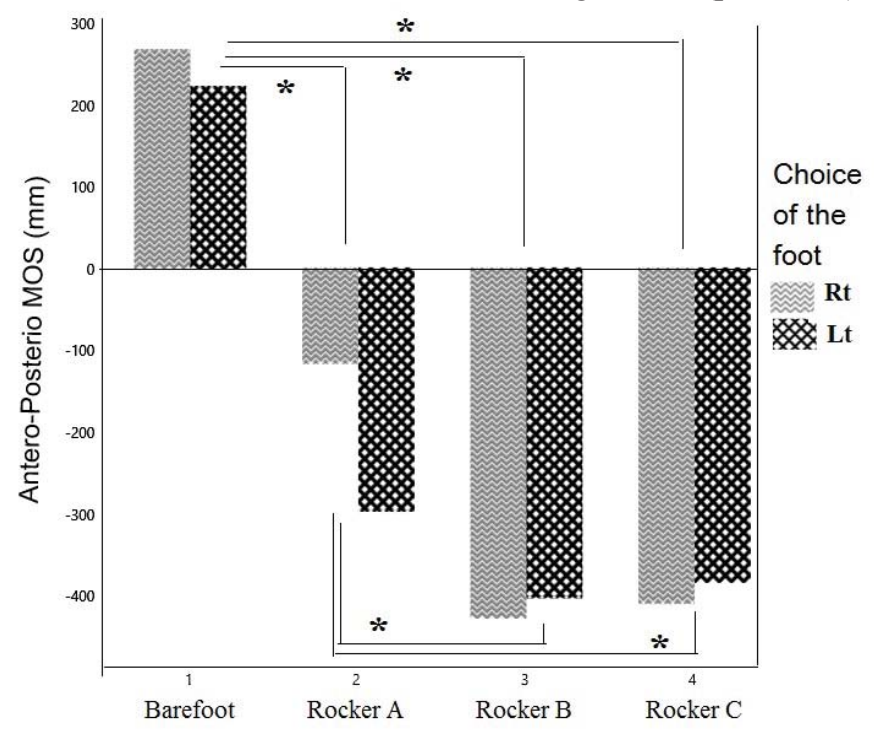

Fig. 2 MOSap with regards to the condition and choice of the foot

Table II The results of MOSml Multiple Comparisons for Condition (Student's t All Pairwise Comparisons)

\begin{tabular}{l|l|l|l|l|}
\hline Condition & -Condition & Difference & $\begin{array}{l}\text { Std } \\
\text { Error }\end{array}$ & Prob>|t| \\
\hline Barefoot & Rocker A & -53.2086 & 51.12493 & 0.3092 \\
\hline Barefoot & Rocker B & -21.2112 & 50.00868 & 0.6755 \\
\hline Barefoot & Rocker C & -50.1583 & 54.31937 & 0.3658 \\
\hline Rocker A & Rocker B & 31.9974 & 46.75867 & 0.5007 \\
\hline Rocker A & Rocker C & 3.0503 & 51.12493 & 0.9530 \\
\hline Rocker B & Rocker C & -28.9471 & 50.00868 & 0.5685 \\
\hline
\end{tabular}

No significant differences were found for each of pairs. 


\section{DISCUSSION}

This study aimed to show how the MOS concept and its equation can be applied to investigate the impact of the rocker outsole shoes on dynamic stability with regard to their specific BOS during walking. Furthermore, we reported statistics for one subject to explain how the results can be interpreted. The negative values of mean MOSap for all three rocker outsole conditions indicated dynamic instability during walking, while this value was positive for barefoot condition and indicated stability. This showed that our subject has been more stable in the antero-posterior direction while walking barefoot compared to walking with all three rocker outsole shoes. This can be due to an increase in antero-posterior COM velocity while walking with the rocker outsole shoes that increased XcoM value and decreased MOS value in anteroposterior direction. The destabilizing effect of both rocker outsoles $\mathrm{B}$ and $\mathrm{C}$ was significantly higher than rocker outsole A. This can be related to increased rocker angle and heel height in rocker $\mathrm{B}$ and $\mathrm{C}$ compared to the rocker $\mathrm{A}$ which can cause more increase in antero-posterior COM velocity thus more decrease in MOSap.

The results also showed that mean MOSml over trials were the same for all conditions but it was significantly different between left and right foot (or between two consecutive steps). This can complement the results found for MOSap. Although the subject was dynamically unstable in antero-posterior direction while walking with all three rocker outsole shoes, she did not experience any falls. It was mentioned earlier that an individual can alter either the positioning of the foot or the COM motion in order to control the MOS. Thus, the subject tried to maintain a constant minimum of MOSml, that was the same between falls.

\section{CONCLUSIONS}

It was shown that MOS equation and its concept is one of the most appropriate methods of investigating dynamic stability while walking with rocker outsole shoes considering their smaller BOS. This pilot study on one female subject with diabetes showed that rocker outsoles can negatively affect antero-posterior dynamic stability. This instability in antero- posterior direction was compensated by either different medio- lateral foot positioning or COM motion in order to preserve a minimum value of MOSml that was the same for all conditions. Thus, the subject remained overall stable without experiencing fall. However, a more conclusive result on how a rocker outsole shoe affects dynamic stability of patients with diabetic neuropathy, will be suggested after fulfilling the ongoing research project. This study is already in progress with an appropriate sample size considering both diabetic and healthy populations, as well as including both genders.

\section{ACKNOWLEDGMENT}

The authors would like to thank Iran University of Medical Sciences for providing Gait Analysis Laboratory, as well as Endocrine Research Centre, Institute of Endocrinology and Metabolism (Firouzgar Hospital), Tehran, Iran.

\section{REFERENCES}

[1] L. Guariguata, D. R. Whiting, I. Hambleton, J. Beagley, U. Linnenkamp, and J. E. Shaw, "Global estimates of diabetes prevalence for 2013 and projections for 2035," Diabetes Res. Clin. Pract., vol. 103, no. 2, pp. 137-149, 2014.

[2] N. Singh, D. G. Armstrong, and B. A. Lipsky, "Preventing foot ulcers in patients with diabetes.," JAMA, vol. 293, no. 2, pp. 217-228, 2005.

[3] C. A. Abbott, A. P. Garrow, A. L. Carrington, J. Morris, E. R. Van Ross, and A. J. Boulton, "Foot ulcer risk is lower in South-Asian and AfricanCaribbean compared with European diabetic patients in the U.K.: The North-West diabetes foot care study," Diabetes Care, vol. 28, no. 8, pp. 1869-1875, 2005.

[4] P. Palumbo and L. I. Melton, "Peripheral vascular disease and diabetes," in Diabetes in America, M. Harris, C. Cowie, M. Stern, and E. Al., Eds. 1995, pp. 401-408.

[5] L. A. Lavery, D. G. Armstrong, R. P. Wunderlich, J. Tredwell, and A. J. M. Boulton, "Predictive value of foot pressure assessment as part of a population-based diabetes disease management program.," Diabetes Care, vol. 26, no. 4, pp. 1069-1073, 2003.

[6] H. M. Rathur and A. J. M. Boulton, "Pathogenesis of foot ulcers and the need for offloading," in Hormone and Metabolic Research, 2005, vol. 37, no. SUPPL. 1.

[7] D. Brown, J. J. Wertsch, G. F. Harris, J. Klein, and D. Janisse, "Effect of rocker soles on plantar pressures," Arch. Phys. Med. Rehabil., vol. 85, no. 1, pp. 81-86, Jan. 2004.

[8] P. S. Schaff and P. R. Cavanagh, "Shoes for the insensitive foot: the effect of a 'rocker bottom' shoe modification on plantar pressure distribution.," Foot Ankle, vol. 11, no. 3, pp. 129-40, Dec. 1990.

[9] C. van Schie, J. S. Ulbrecht, M. B. Becker, and P. R. Cavanagh, "Design criteria for rigid rocker shoes.," Foot Ankle Int., vol. 21, no. 10, pp. 833-44, Oct. 2000.

[10] G. D. Fulk, C. J. Robinson, S. Mondal, C. M. Storey, and A. M. Hollister, "The effects of diabetes and/or peripheral neuropathy in detecting short postural perturbations in mature adults," J. Neuroeng. Rehabil., vol. 7, no. 1, p. 44, Sep. 2010.

[11] S. Morrison, S. R. Colberg, H. K. Parson, and A. I. Vinik, "Relation between risk of falling and postural sway complexity in diabetes.," Gait Posture, vol. 35, no. 4, pp. 662-8, Apr. 2012.

[12] R. Yamamoto, T. Kinoshita, T. Momoki, T. Arai, A. Okamura, K. Hirao, and H. Sekihara, "Postural sway and diabetic peripheral neuropathy.," Diabetes Res. Clin. Pract., vol. 52, no. 3, pp. 213-221, 2001.

[13] I. C. N. Sacco, A. N. Hamamoto, A. N. Onodera, A. A. Gomes, H. A. Weiderpass, C. G. F. Pachi, J. F. Yamamoto, and V. von Tscharner, "Motor strategy patterns study of diabetic neuropathic individuals while walking. A wavelet approach.," J. Biomech., vol. 47, no. 10, pp. 247582, Jul. 2014.

[14] I. C. N. Sacco, A. P. Picon, D. O. Macedo, M. K. Butugan, R. Watari, and C. D. Sartor, "Alterations in the lower limb joint moments precede the peripheral neuropathy diagnosis in diabetes patients.," Diabetes Technol. Ther., vol. 17, no. 6, pp. 405-12, Jun. 2015.

[15] H. S. Zhu, J. J. Wertsch, G. F. Harris, J. D. Loftsgaarden, and M. B. Price, "Foot pressure distribution during walking and shuffling.," Arch. Phys. Med. Rehabil., vol. 72, no. 6, pp. 390-397, 1991.

Predisposing and Situational Risk Factors to Serious Fall Injuries," J. Am. Geriatr. Soc., vol. 43, no. 11, pp. 1207-1213, Nov. 1995.

[16] O. Y. Kwon and M. J. Mueller, "Walking patterns used to reduce forefoot plantar pressures in people with diabetic neuropathies.," Phys. Ther., vol. 81, no. 2, pp. 828-35, Feb. 2001.

[17] S. Tesfaye, "Recent advances in the management of diabetic distal symmetrical polyneuropathy," Journal of Diabetes Investigation, vol. 2, no. 1. pp. 33-42, 2011.

[18] C. T. Bonnet and C. Ray, "Peripheral neuropathy may not be the only fundamental reason explaining increased sway in diabetic individuals.," Clin. Biomech. (Bristol, Avon), vol. 26, no. 7, pp. 699-706, Aug. 2011.

[19] J. K. Richardson and E. A. Hurvitz, "Peripheral neuropathy: a true risk factor for falls.," J. Gerontol. A. Biol. Sci. Med. Sci., vol. 50, no. 4, pp. M211-M215, 1995. 
[20] L. M. Tilling, K. Darawil, and M. Britton, "Falls as a complication of diabetes mellitus in older people," J. Diabetes Complications, vol. 20, no. 3, pp. 158-162, May 2006

[21] B. C. Albright and W. M. Woodhull-Smith, "Rocker bottom soles alter the postural response to backward translation during stance.," Gait Posture, vol. 30, no. 1, pp. 45-9, Jul. 2009.

[22] B. Ghomian, M. Kamyab, H. Jafari, M. Khamseh, and A. Healy, "Rocker outsole shoe is not a threat to postural stability in patients with diabetic neuropathy.," Prosthet. Orthot. Int., vol. 40, no. 2, pp. 224-30, Apr. 2016.

[23] M. Lumeau, M. Lempereur, and O. Remy-Neris, "Assessment of the rocker sole shoes on postural stability in diabetic patients with distal sensory neuropathy," Comput. Methods Biomech. Biomed. Engin., 2015.

[24] M. E. Tinetti, J. T. Doucette, and E. B. Claus, "The Contribution of Predisposing and Situational Risk Factors to Serious Fall Injuries," J. Am. Geriatr. Soc., vol. 43, no. 11, pp. 1207-1213, Nov. 1995.

[25] N. Niino, S. Tsuzuku, F. Ando, and H. Shimokata, "Frequencies and circumstances of falls in the National Institute for Longevity Sciences, Longitudinal Study of Aging (NILS-LSA).," J. Epidemiol., vol. 10, no. 1 Suppl, pp. S90-4, Apr. 2000.
[26] S. Hutchins, P. Bowker, N. Geary, and J. Richards, "The biomechanics and clinical efficacy of footwear adapted with rocker profiles--evidence in the literature.," Foot (Edinb)., vol. 19, no. 3, pp. 165-70, Sep. 2009.

[27] F. Benvenuti, R. Mecacci, I. Gineprari, S. Bandinelli, E. Benvenuti, L. Ferrucci, A. Baroni, M. Rabuffetti, M. Hallett, J. M. Dambrosia, and S. J. Stanhope, "Kinematic characteristics of standing disequilibrium: reliability and validity of a posturographic protocol.," Arch. Phys. Med. Rehabil., vol. 80, no. 3, pp. 278-87, Mar. 1999.

[28] A. L. Hof, M. G. J. Gazendam, and W. E. Sinke, "The condition for dynamic stability.," J. Biomech., vol. 38, no. 1, pp. 1-8, Jan. 2005.

[29] A. L. Hof, "The 'extrapolated center of mass' concept suggests a simple control of balance in walking.," Hum. Mov. Sci., vol. 27, no. 1, pp. 11225, Feb. 2008.

[30] J. D. Chapman, S. Preece, B. Braunstein, A. Höhne, C. J. Nester, P. Brueggemann, and S. Hutchins, "Effect of rocker shoe design features on forefoot plantar pressures in people with and without diabetes.," Clin. Biomech. (Bristol, Avon), vol. 28, no. 6, pp. 679-85, Jul. 2013.

[31] P. M. McAndrew Young and J. B. Dingwell, "Voluntary changes in step width and step length during human walking affect dynamic margins of stability.," Gait Posture, vol. 36, no. 2, pp. 219-24, Jun. 2012. 